Marquette University

e-Publications@Marquette

Biomedical Engineering Faculty Research and

Publications

Biomedical Engineering, Department of

11-1-2009

\title{
Comparison of Cumulative Planimetry versus Manual Dissection to Assess Experimental Infarct Size in Isolated Hearts
}

\author{
Matthias L. Riess \\ Medical College of Wisconsin \\ Samhita S. Rhodes \\ Padnos College of Engineering and Computing \\ David F. Stowe \\ Marquette University \\ Mohammed Aldakkak \\ Medical College of Wisconsin \\ Amadou K.S. Camara \\ Medical College of Wisconsin
}

Accepted version. Journal of Pharmacological and Toxicological Methods, Vol. 60, No. 3 (November/ December 2009): 275-280. DOI. (C) 2009 Elsevier. Used with permission. 


\title{
Comparison of Cumulative Planimetry versus Manual Dissection to Assess Experimental Infarct Size in Isolated Hearts
}

\author{
Matthias L. Riess \\ Anesthesiology Research Laboratories, Departments of \\ Anesthesiology and Physiology, Medical College of Wisconsin, \\ and VA Medical Center \\ Milwaukee, WI \\ Samhita S. Rhodes \\ School of Engineering, Padnos College of Engineering and \\ Computing \\ Grand Rapids, MI \\ David F. Stowe \\ Anesthesiology Research Laboratories, Departments of \\ Anesthesiology and Physiology, Cardiovascular Research Center, \\ Medical College of Wisconsin and VA Medical Center, and \\ Department of Biomedical Engineering, Marquette University, \\ Milwaukee, WI \\ Mohammed Aldakkak \\ Anesthesiology Research Laboratories, Department of \\ Anesthesiology, Medical College of Wisconsin \\ Milwaukee, WI \\ Amadou K.S. Camara \\ Anesthesiology Research Laboratories, Department of \\ Anesthesiology, Medical College of Wisconsin, \\ Milwaukee, WI
}


NOT THE PUBLISHED VERSION; this is the author's final, peer-reviewed manuscript. The published version may be accessed by following the link in the citation at the bottom of the page.

\section{Abstract}

\section{Introduction}

Infarct size (IS) is an important variable to estimate cardiac ischemia/reperfusion injury in animal models. Triphenyltetrazoliumchloride (TTC) stains viable cells red while leaving infarcted cells unstained. To quantify IS, infarcted and non-infarcted tissue is often manually dissected and weighed (IS-DW). An alternative is to measure infarcted areas by cumulative planimetry (IS-CP).

\section{Methods}

We prospectively compared these two methods in 141 Langendorffprepared guinea pig hearts $(1.44 \pm 0.02 \mathrm{~g})$ that were part of different studies on mechanisms of cardioprotection. Hearts were perfused with Krebs-Ringer's and subjected to 30 min global ischemia after various cardioprotective treatments. Two hours after reperfusion hearts were cut into 6-7 transverse sections $\left(3 \mathrm{~mm}\right.$ ) and stained for $5 \mathrm{~min}$ in $1 \% \mathrm{TTC}$ and $0.1 \mathrm{M} \mathrm{KH}_{2} \mathrm{PO}_{4}$ buffer $\left(\mathrm{pH} 7.4,38^{\circ} \mathrm{C}\right)$. Each slice was first scanned and its infarcted area measured with Image 1.62 software (NIH). Infarctions in individual slices of each heart were averaged (IS-CP) on the basis of their weight. After scanning, IS-DW was determined by careful manual dissection of infarcted from noninfarcted tissue and measuring their respective total weight.

\section{Results}

We found limited tissue permeation of $\pi \mathrm{TC}$ in relation to the slice thickness leaving tissue in the center unstained, as well as significant crosscontamination of stained vs. unstained tissue after manual dissection. IS-CP and IS-DW ranged from 6.0 to $73.1 \%$ and 19.4 to $70.5 \%$, respectively, and correlated as follows: IS-DW $=(27.6 \pm 1.4)+(0.518 \pm 0.038) \cdot$ IS-CP; $r=$ 0.75 (Pearson), $P<0.001$. In addition, IS-CP correlated better with return of function after reperfusion like developed left ventricular pressure, contractility and relaxation, and myocardial oxygen consumption.

\section{Discussion}

Despite a good correlation between both methods, limited tissue permeation by TTC diffusion and limited precision in the ability to manually dissect stained from unstained tissue leads to an overestimation of infarct size by dissection and weighing compared to cumulative planimetry. 
NOT THE PUBLISHED VERSION; this is the author's final, peer-reviewed manuscript. The published version may be accessed by following the link in the citation at the bottom of the page.

Keywords: cumulative planimetry, guinea pig, heart, infarct size, methods, myocardium, planimetry, staining, triphenyltetrazoliumchloride, TTC

\section{Introduction}

Considerable investigative efforts over recent decades have focused on the study of ischemia/reperfusion (I/R) injury in general, and cardioprotective strategies in particular. Indeed, the study of preand post-conditioning alone now leads to several hundred articles per year in the peer-reviewed literature. The vast majority of these investigations have relied on isolated hearts or intact animal models (Hausenloy \& Yellon, 2007). I/R is usually applied before or after one or more interventions, and the degree of cardioprotection is subsequently assessed by measuring and comparing the various degrees of myocardial infarction.

Therefore, infarct size (IS) measurement has become pivotal in several areas of cardiac research, to the extent that many investigations are ultimately based mainly or solely on this variable. However, use of different methods by various investigators makes comparisons among laboratories challenging. 2,3,5triphenyltetrazolium chloride (TTC) staining is a widely used and accepted method to delineate infarcted from non-infarcted tissue and is well correlated with more traditional staining procedures (Fishbein et al., 1981; Khalil et al., 2006). TTC itself is colorless in solution, but is reduced by dehydrogenases of functioning mitochondria to yield a brick red formazan (Altman, 1976). Infarcted tissue remains unstained and can be recognized without microscopic examination. Nevertheless, there is considerable variation in how TTC is used to stain the tissue perfusion into the coronaries before slicing or diffusion from the surface after slicing - and in how the staining results are converted to quantify IS. Some investigators, for example, manually dissect the tissue and separately weigh infarcted and non-infarcted tissue (Chiari et al., 2004; Stowe, Camara, Heisner, Aldakkak \& Harder, 2007). Others use a computer-based method, in which cross-sectional images of heart slices are made, and IS is measured by color analysis using specialized software (Fishbein et al., 1981). This latter method, however, only measures two-dimensional surface staining in a finite number of slices, and measurement of infarction in the third dimension 
is solely a factor of the number of slices. In addition, tissue penetration by TTC diffusion may be incomplete, thus it is possible that the planimetric method misses valuable information.

To our knowledge, no effort has been made to compare the image-based planimetric technique to the physical dissection and weighing of the tissue after TTC staining. We used Langendorffprepared guinea pig hearts that were part of different studies on mechanisms of cardioprotection where hearts were subjected to various cardioprotective strategies followed by I/R. These hearts were studied prospectively to contrast the results of the two different methods in discriminating less infarcted from more infarcted myocardium. In each heart, IS was measured first by cumulative planimetry, then by manual dissection.

\section{Methods}

\subsection{Statement on Use and Care of Animals}

All investigations conformed to the Guide for the Care and Use of Laboratory Animals (US National Institutes of Health No. 85-23, revised 1996) and were approved by the Institutional Animal Care and Use Committee (Medical College of Wisconsin, Milwaukee, WI, U.S.A.). Thirty mg of ketamine and 1,000 units of heparin were injected intraperitoneally into 143 albino English short-haired guinea pigs (weight 250-300 g) of either sex that were, with the exception of two hearts, part of different studies on cardioprotection (Riess et al., 2002; Riess, Camara, Kevin, An \& Stowe, 2004; Riess et al., 2003). Animals were decapitated 15 min later when unresponsive to noxious stimulation.

\subsection{Langendorff Heart Preparation}

Our methods have been described previously (Riess et al., 2002; Riess, Camara, Kevin, An \& Stowe, 2004; Riess et al., 2003). After thoracotomy, the aorta was cannulated distal to the aortic valve, and the heart was immediately perfused retrograde with $4^{\circ} \mathrm{C}$ cold oxygenated Krebs-Ringer solution. The inferior and superior venae cavae were ligated, and the heart was rapidly excised (wet weight 
$1.44 \pm 0.02 \mathrm{~g})$. After cannulation of the pulmonary artery to collect the coronary effluent, the heart was placed in the support system and perfused at $55 \mathrm{mmHg}$ at $37^{\circ} \mathrm{C}$. The Krebs-Ringer perfusate was equilibrated with $\sim 97 \% \mathrm{O}_{2}$ and $\sim 3 \% \mathrm{CO}_{2}$ to maintain a constant perfusion $\mathrm{pH}$ of $7.40 \pm 0.01$ with a carbon dioxide partial pressure $\left(\mathrm{pCO}_{2}\right)$ of $25 \pm 2 \mathrm{mmHg}$ and an oxygen partial pressure $\left(\mathrm{pO}_{2}\right)$ of 570 $\pm 10 \mathrm{mmHg}$. The perfusate was filtered ( $5-\mu \mathrm{m}$ pore size) in-line and had the following calculated composition (nonionized): $138 \mathrm{mM} \mathrm{Na}^{+}$, $4.5 \mathrm{mM} \mathrm{K}^{+}, 1.2 \mathrm{mM} \mathrm{Mg}^{2+}, 2.5 \mathrm{mM} \mathrm{Ca}^{2+}, 134 \mathrm{mM} \mathrm{Cl}^{-}, 14.5 \mathrm{mM} \mathrm{HCO}_{3}{ }^{-}$, $1.2 \mathrm{mM} \mathrm{H}_{2} \mathrm{PO}_{4}^{-}$, $11.5 \mathrm{mM}$ glucose, $2 \mathrm{mM}$ pyruvate, $16 \mathrm{mM}$ mannitol, $0.1 \mathrm{mM}$ probenecid, $0.05 \mathrm{mM}$ EDTA, and $5 \mathrm{U} / \mathrm{I}$ insulin.

Left ventricular pressure (LVP) was measured isovolumetrically with a saline-filled latex balloon inserted into the left ventricle through a cut in the left atrium. At the beginning of the experiment the balloon volume was adjusted to achieve a diastolic LVP of $0 \mathrm{mmHg}$, so that any subsequent increase in diastolic LVP reflected an increase in left ventricular wall stiffness, or diastolic contracture. Characteristic data from LVP were as follows: developed (systolicdiastolic) LVP, and the maximal and minimal first derivatives of LVP (dLVP/dt $\max$ and $\mathrm{dLVP} / \mathrm{dt}_{\min }$ ) as indices of contractility and relaxation, respectively. Coronary flow (CF) was measured at constant temperature and perfusion pressure by an ultrasonic flowmeter (Transonic T106X, Ithaca, NY) placed directly into the aortic inflow line. Coronary inflow (a) and venous $(\mathrm{v}) \mathrm{pO}_{2}, \mathrm{pH}$, and $\mathrm{pCO}_{2}$ were measured off-line with an intermittently self-calibrating analyzer system (Radiometer Copenhagen ABL 505, Copenhagen, Denmark). $\mathrm{pO}_{2} \mathrm{v}$ tension was also measured continuously on-line with an $\mathrm{O}_{2}$ Clark type electrode (model 203B; Instech, Plymouth Meeting, PA). Myocardial oxygen consumption $\left(\mathrm{MVO}_{2}\right)$ was calculated as $\mathrm{CF}$ - heart wet weight ${ }^{-1} \bullet\left(\mathrm{pO}_{2} \mathrm{a}\right.$ $\left.-\mathrm{pO}_{2} \mathrm{v}\right) \cdot 24 \mu \mathrm{l} \mathrm{O}_{2} / \mathrm{ml}$ at $760 \mathrm{mmHg}$. The degree of functional cardioprotection was assessed as \% return of LVP, dLVP/dt $\mathrm{dax}_{\text {, }}$ $\mathrm{dLVP} / \mathrm{dt}_{\min }$ and $\mathrm{MVO}_{2}$, respectively, at 120 min reperfusion compared to their respected baseline values before I/R.

\subsection{Cardioprotective Strategies}

Different levels of myocardial infarction were obtained by applying various strategies of cardioprotection such as ischemic 
preconditioning (IPC) (Murry, Jennings \& Reimer, 1986), anesthetic preconditioning (APC) (Kersten, Schmeling, Pagel, Gross \& Warltier, 1997) or hypothermia (Camara, Riess, Kevin, Novalija \& Stowe, 2004). Time control hearts (CON; $n=20$ ) were perfused for $190 \mathrm{~min}$ and not subjected to I/R. All other hearts underwent 30 min of global no-flow ischemia followed by 120 min of reperfusion. Ischemic control hearts (ISC; $n=68$ ) were not subjected to cardioprotection. IPC $(n=8)$ was achieved by two times 5 min of global ischemia with 5 min reperfusion between the two IPC episodes and 20 min between the second IPC episode and index ischemia (Riess et al., 2002). APC was achieved by exposure to $0.5 \pm 0.1 \mathrm{mM}(\mathrm{n}=8)$ or $1.3 \pm 0.1 \mathrm{mM}(\mathrm{n}=22)$ sevoflurane (Abbott Laboratories, Chicago, IL) for 15 min ending 20 min before index ischemia (Riess et al., 2002; Riess et al., 2003). Hypothermic cardioprotection $(n=15)$ was achieved by cooling from 37 to $17^{\circ} \mathrm{C} 20$ min before index ischemia and rewarming on reperfusion (Riess, Camara, Kevin, An \& Stowe, 2004). At the end of each experiment, hearts were removed from the perfusion apparatus and IS was determined as described below. Two additional hearts were used to specifically investigate the depth of TTC permeation by diffusion from the slices' surface immediately after removal from the chest.

\subsection{Assessment of Infarct Size}

\subsubsection{TTC Staining}

At the end of 120 min of reperfusion, hearts were quickly removed and weighed. Atria were discarded and ventricles were cut into 6 to 7 uniform transverse slices of $3 \mathrm{~mm}$ thickness using a rat heart matrix. Slices were immediately stained by diffusion of $1 \%$ TTC (Sigma; St. Louis, $\mathrm{MO}$ ) in $0.1 \mathrm{M} \mathrm{KH}_{2} \mathrm{PO}_{4}$ buffer $\left(\mathrm{pH} 7.4,38^{\circ} \mathrm{C}\right.$ ) for 5 min. TTC stains viable tissue red, indicating the presence of a formazan precipitate that results from the reduction of TTC by dehydrogenases present in viable tissue only (Altman, 1976; Fishbein et al., 1981).

\subsubsection{Scanner-Based Image Acquisition}

Slices were individually weighed and positioned on a transparent sheet protector with their apical surface facing upwards. The sheet and 
slices were then placed on the scanning surface of a color scanner (ScanJet 6300C, Hewlett-Packard, Palo Alto, CA) connected to a personal computer (Dimension L866r, Dell, Round Rock, TX) and controlled with the appropriate software (HP Precision Scan Pro, Hewlett-Packard). The scanner was set to acquire 24-bit color images at 1200 pixels per inch image resolution. The resulting images were saved as uncompressed tagged image files. Figs. $1 A$ and $B$ show examples from two different types of hearts representing different magnitudes of TTC staining.

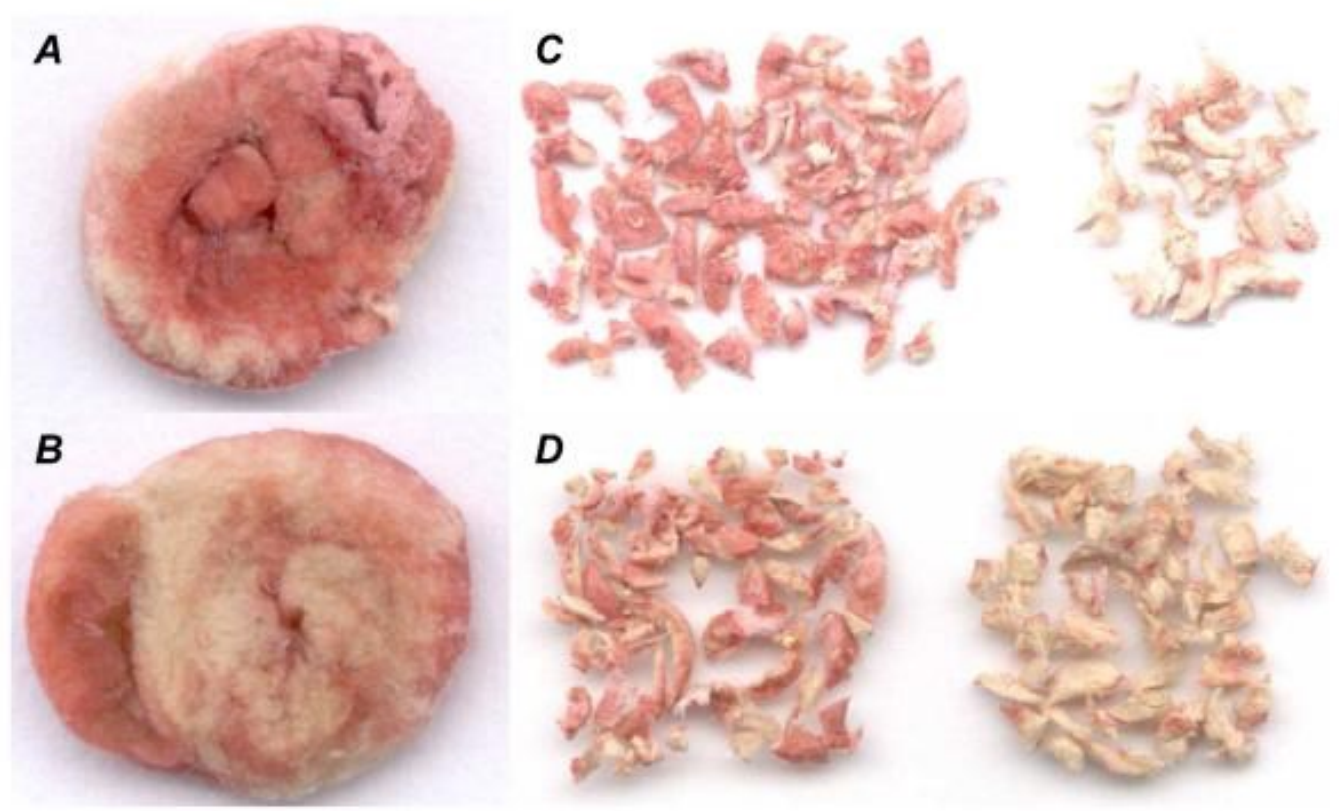

Figure 1 shows scanned sample slices from two different hearts after TTC staining: a nonischemic time control heart (panel $A$ ) with $16.6 \%$ infarcted area, and an ischemic control heart (panel $B$ ) with $56.9 \%$ infarcted area as measured by planimetry. After imaging, the TTC-stained and unstained tissue was carefully separated by manual dissection. Panel $C$ shows the stained vs. unstained tissue of the non-ischemic time control heart from panel $A$, panel $D$ shows the stained vs. unstained tissue of the ischemic time control heart from panel $B$. Please note the crosscontamination of stained and unstained tissue despite careful dissection.

\subsubsection{Infarct Size Assessment by Cumulative Planimetry}

Images were analyzed manually with a computer mouse by a person blinded to the experimental protocol using public domain image processing software (Image 1.62; National Institutes of Health, Bethesda, MD). The (sum of the) infarcted area(s) $\left(I A_{i}\right)$ of an 
individual slice (i) was measured and divided by the measured total area of that slice $\left(T A_{i}\right)$ to obtain the fraction of infarction of the slice. Results from individual (i) slices of each heart were averaged on the basis of their weight $\left(w t_{i}\right)$ to calculate the total ventricular IS (IS-CP) for each heart in percent by using the following formula:

$$
\begin{gathered}
\mathrm{IS}-\mathrm{CP}(\%)=\left[\left(\mathrm{IA}_{1} / \mathrm{TA}_{1} \bullet w \mathrm{t}_{1}\right)+\left(\mathrm{IA}_{2} / \mathrm{TA}_{2} \bullet w \mathrm{t}_{2}\right)+\ldots+\left(\mathrm{IA}_{\mathrm{i}} / \mathrm{TA}_{\mathrm{i}} \bullet\right.\right. \\
\left.\left.w t_{i}\right)\right] /\left(\mathrm{wt}_{1}+\mathrm{wt}_{2}+\ldots+\mathrm{wt}_{\mathrm{i}}\right)
\end{gathered}
$$

\subsubsection{Infarct Size Assessment by Dissection and Weighing}

After imaging, the red-stained and unstained tissue of all slices of each individual heart was physically dissected under an illuminated magnifier ( 3 diopter, VWR Scientific Products, West Chester, PA) by another person blinded to the experimental protocol. Total weights of stained and unstained tissue of each individual heart were measured to determine the total ventricular IS (IS-DW) for each heart in percent. Figs $1 C$ and $D$ show examples from the two different hearts corresponding to Fig $1 A$ and $B$, respectively.

\subsection{Statistics}

All data are expressed as means \pm standard errors of the means (SEM). Regression and correlation analysis (Pearson) was used to assess the relationship between IS-CP and IS-DW as well as between either method and other variables on reperfusion. Differences were considered statistically significant $(*)$ when $P<0.05$ (two-tailed).

\section{Results}

The 141 individual IS pairs are shown in Fig 2. IS-CP and IS-DW ranged from 6.0 to $73.1 \%$ and 19.4 to $70.5 \%$, respectively. Regression analysis revealed a linear relation between the two different methods: IS-DW $=(27.6 \pm 1.4)+(0.518 \pm 0.038) \bullet$ IS-CP; $r=$ $0.75, P<0.001$. 


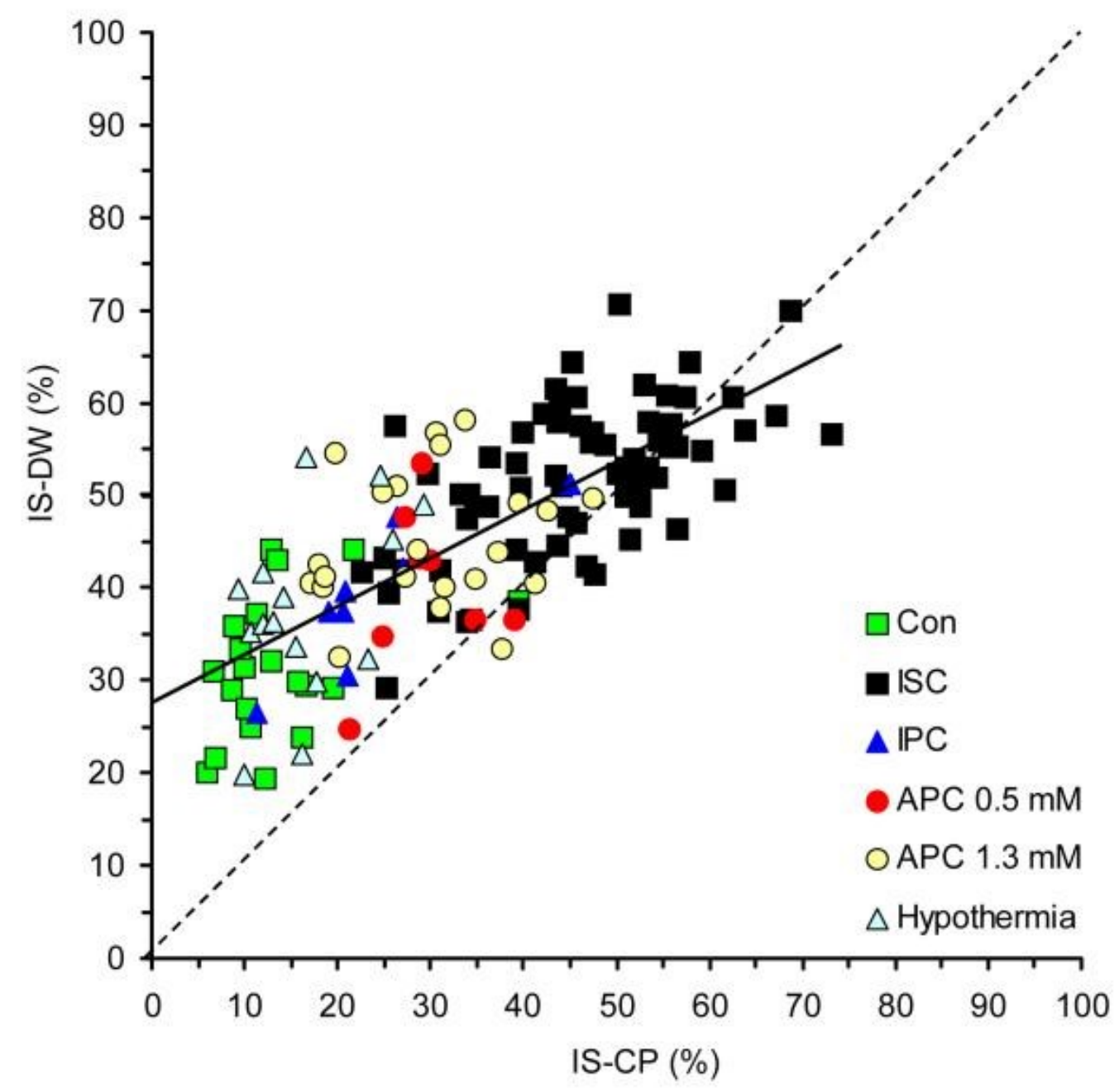

Figure 2 shows the 141 individual pairs of infarct size measurements in \% with the two different methods: IS-CP by cumulative planimetry and IS-DW by dissection and weighing. Hearts from different cardioprotective strategies (see text) are depicted by different symbols. Regression analysis revealed a linear relation and good correlation between the two different methods for all hearts: IS-DW $=(27.6 \pm 1.4)+$ $(0.518 \pm 0.038) \bullet$ IS-CP; $r=0.75, P<0.001$. Please note the deviation of this relation (continuous line) with an intercept of $27.6 \% *$ and a slope of only $0.52 *$ from the line of identity (broken line) with an ideal intercept of $0 \%$ and an ideal slope of 1.00 . The two lines cross at the $57.2 \%$ mark.

Longitudinal sections of transverse slices from the additional two non-ischemic hearts taken immediately after surgery showed that despite a good coloration on the surface (Fig $3 A$ and $B$ ) the staining depth (x) of TTC reached only $\sim 0.2$ to $0.5 \mathrm{~mm}$ (Fig $3 C$ and $D$ ). 

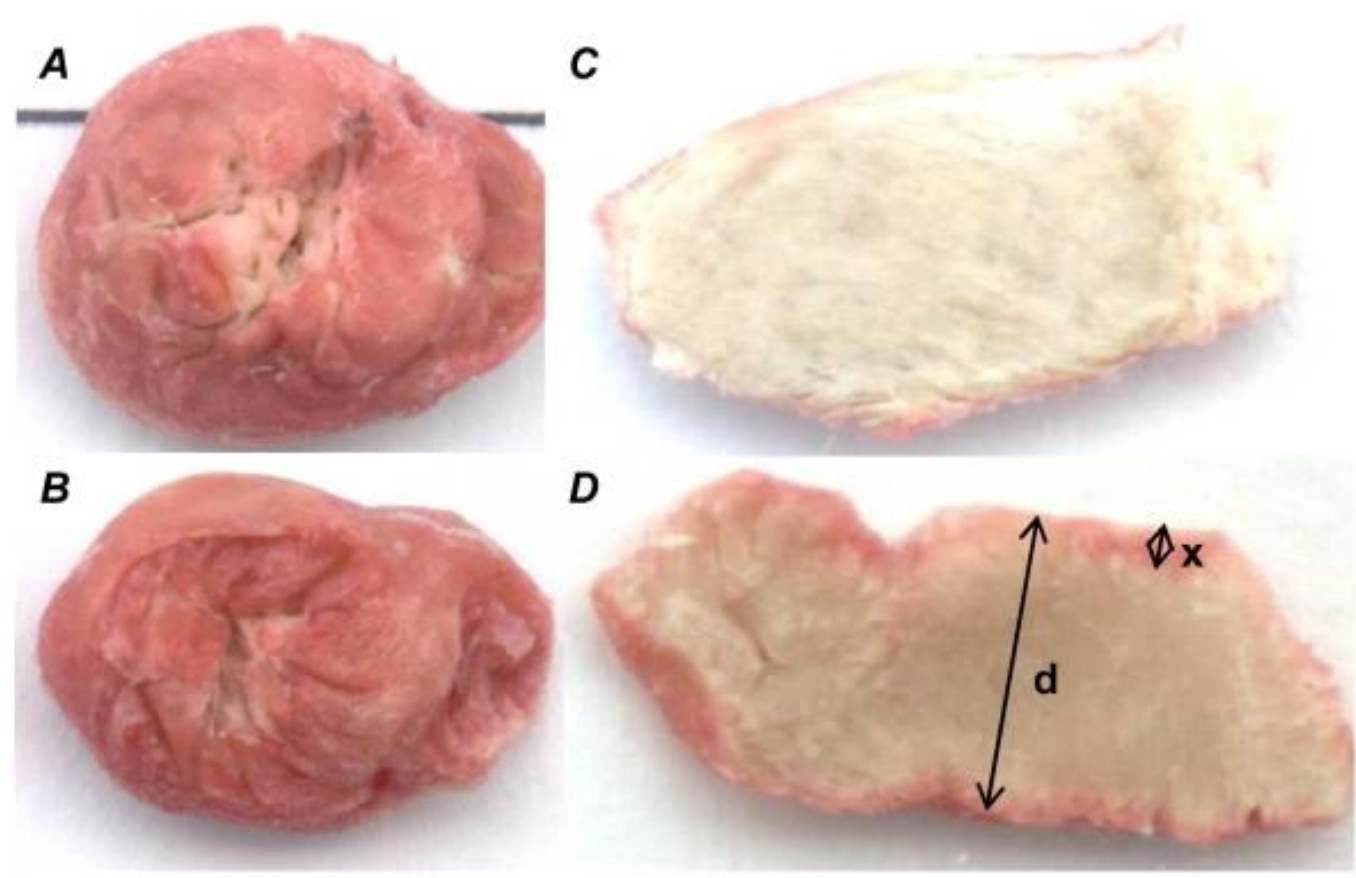

Figure 3 shows longitudinal sections of transverse slices (panels $C$ and $D$ ) from two additional non-ischemic hearts stained immediately after cannulation. Despite a good coloration by $\Pi T C$ staining on the surface (panels $A$ and $B$ ) the staining depth (x) reached only $\sim 0.2 \mathrm{~mm}$ (panel $C$ ) and $0.5 \mathrm{~mm}$ (panel $D$ ) in relation to the slice's thickness $d$, leaving $d-2 x$ unstained.

Furthermore, stepwise regression analysis between IS-CP and IS-DW as independent variables and \%dLVP/dt $t_{\max }, \% d L V P / d t_{\min }$, $\%$ devLVP, and $\% \mathrm{MVO}_{2}$ as dependent variables showed that IS-CP did whereas IS-DW did not correlate significantly with the return of mechanical and metabolic function at 120 min reperfusion (Fig $4 A$ to $D)$. 

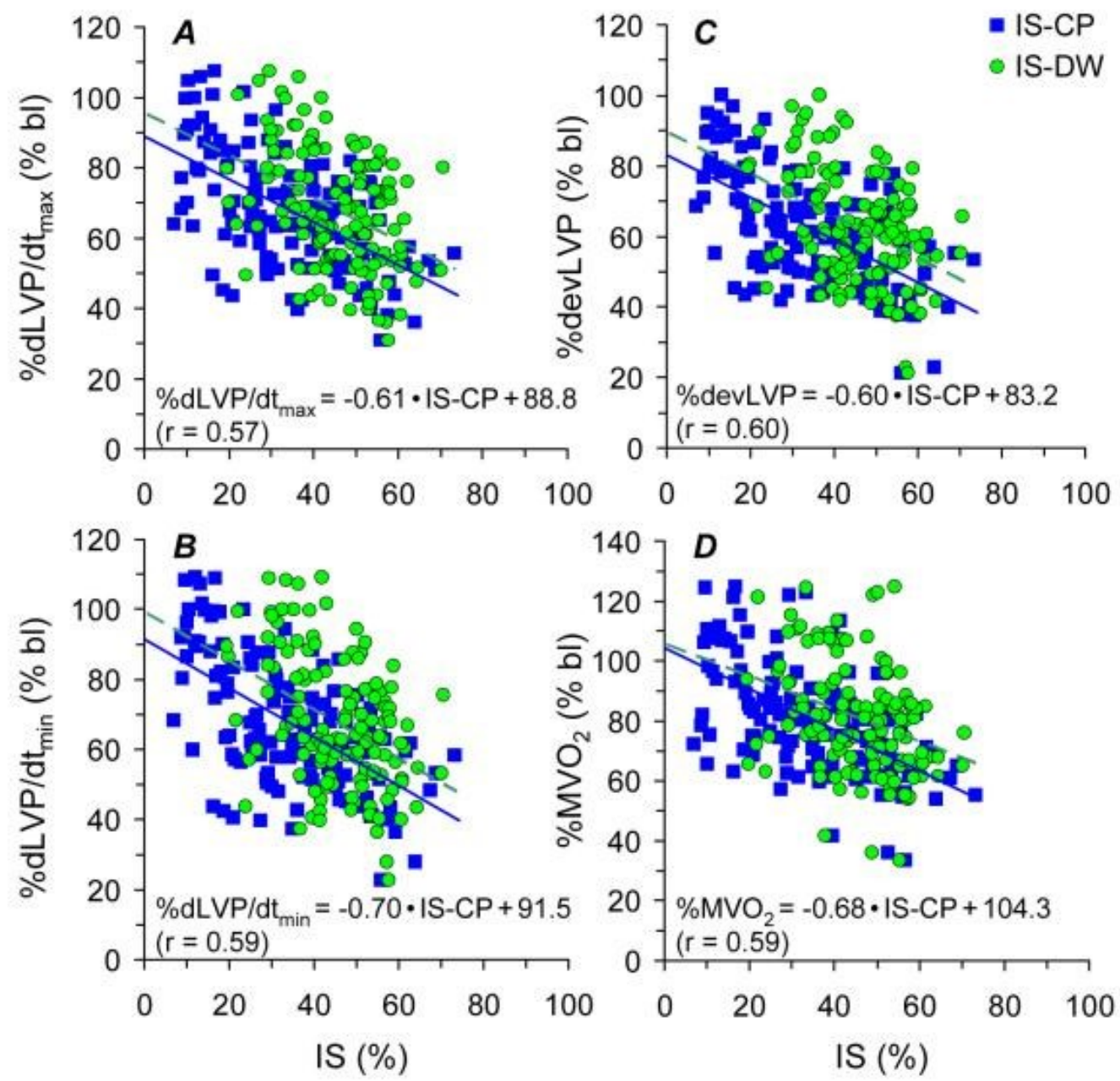

Figure 4 infarct sizes (IS) in \% measured by cumulative planimetry (IS-CP; blue squares, continuous line) and by dissection and weighing (IS-DW; green circles, broken line) are plotted against return of contractility (\%dLVP/dt max; panel $A$ ), relaxation (\%dLVP/dt $\min$; panel $B$ ), developed left ventricular pressure (\%devLVP; panel $C$ ), and myocardial oxygen consumption $\left(\% \mathrm{MVO}_{2}\right.$; panel $D$ ) at $120 \mathrm{~min}$ reperfusion as \% of baseline (bl) before ischemia. Stepwise regression analysis between IS-CP and IS-DW as independent variables and \%dLVP/dt $t_{\max }, \% d L V P / d t_{\min }$, $\%$ devLVP, and $\% \mathrm{MVO}_{2}$ as dependent variables showed that IS-CP did whereas IS-DW did not correlate significantly with the return of mechanical and metabolic function on reperfusion.

\section{Discussion}

Assessment of the quantity of I/R injury and cardioprotective strategies in animal experiments often depends critically on the reliable measurement of IS as the gold standard to assess tissue

Journal of Pharmacological and Toxicological Methods, Vol. 60, No. 3 (November/December 2009): pg. 275-280. DOI. This article is (C Elsevier and permission has been granted for this version to appear in e-Publications@Marquette. Elsevier does not grant permission for this article to be further copied/distributed or hosted elsewhere without the express permission from Elsevier. 
viability. TTC staining is an easy and well-established method to delineate infarcted from non-infarcted tissue (Fishbein et al., 1981; Khalil et al., 2006). Our study compares two different methods to quantify IS after global I/R in guinea pig isolated hearts, cumulative planimetry and manual dissection and weighing following TTC staining of freshly removed and sliced hearts. We found a highly significant linear relation and good correlation between both methods, and therefore the two methods may - at first - appear to be equally useful. A closer examination, however, reveals a) a significant intercept and b) a slope significantly lower than 1.0 in the relation between dissection/weighing and cumulative planimetry.

Both methods have their individual advantages and disadvantages. Manually separating red from white areas on a largely magnified two-dimensional virtual image (Fig $1 A$ and $B$ ) by using a computer mouse is reasonably exact, reproducible, and reversible in case of errors; moreover, within limitations it can even be done operator-independent by automated image analysis (Goldlust, Paczynski, He, Hsu \& Goldberg, 1996; Hindman, Dexter, Subieta, Smith \& Cutkomp, 1999). In contrast, physical dissection of very small and loose tissue pieces goes along with a significant contamination of red with white tissue mass and vice versa (Fig $1 C$ and $D$ ). While this is expected to cancel out when the stained and unstained tissue masses are equal, i.e. $50 \%$, fractions of stained vs. unstained tissue higher or lower than 50\%, however, will lead to a lower discrimination: low IS will be measured falsely higher, high IS will be measured falsely lower, and more so the less precision $(p ; 0<p<1)$ is used during the manual dissection process. The expected relation between IS-DW and IS-CP would therefore be: IS-DW $\approx 50 \bullet(1-\mathrm{p})+\mathrm{p} \bullet$ ISCP with $\mathrm{p} \rightarrow$ $0:$ IS-DW $\approx 50 \%$ and with $\mathrm{p} \rightarrow 1:$ IS-DW $\approx$ IS-CP (Fig $5 A$ ). 


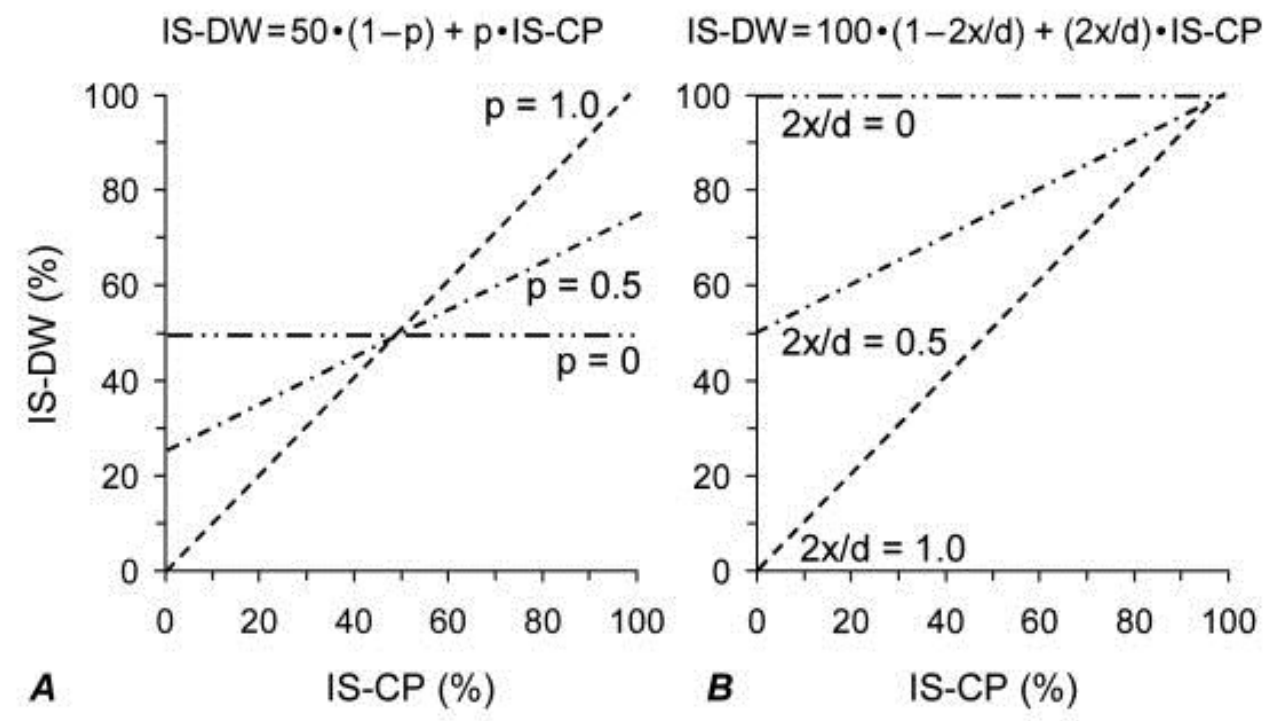

Figure 5 panel $A$ : Manual dissection of stained from unstained tissue pieces is associated with an unavoidable cross-contamination (also see Fig 1 ); this is expected to cancel out when the stained and unstained tissue masses are equal, but different fractions will lead to lower discrimination, more so the less precision $(p ; 0<p<1)$ is used during the manual dissection process. The expected relation between both methods is: IS-DW $\approx 50 \bullet(1-\mathrm{p})+\mathrm{p} \bullet$ IS-CP with $\mathrm{p} \rightarrow 0:$ IS-DW $\approx 50 \%$ and with $\mathrm{p}$ $\rightarrow 1:$ IS-DW $\approx$ IS-CP. All lines meet at the $50 \%$ mark. Panel $B$ : Because of limited tissue permeation $(x)$ of TTC in a given slice with the thickness $d$, the dissection and weighing method will overestimate the actual infarct size, depending on the ratio of $x$ and $d$. The expected relation between both methods is: IS-DW $\approx 100 \bullet(1-2 x / d)+$ $(2 x / d) \bullet$ IS-CP, with $2 x / d \rightarrow 0:$ IS-DW $\approx 100 \%$ (no tissue permeation) and with $2 x / d$ $\rightarrow 1:$ IS-DW $\approx$ ISCP (full tissue permeation). All lines meet at the $100 \%$ mark.

While dissection and weighing is a three-dimensional method, the resolution of cumulative planimetry in the longitudinal axis, on the other hand, depends on the number of slices made in the longitudinal direction. This, however, is limited by the heart size of a given animal and the minimally required thickness of each slice which depends on the tissue consistence of the fresh myocardium in a given species. The planimetric approach therefore functions on the assumption of an identical percentage of infarction throughout the different planes in the longitudinal direction of each individual slice.

Longitudinal sections of slices from hearts not subjected to I/R (Fig 3 ) in our study revealed rather limited tissue permeation ( $x$ ) from either surface towards the center of the slice, thus leaving a considerable distance between the surfaces $(d-2 x)$ unstained 
regardless of its viability. Although these slices would be expected to have close to no infarction which was confirmed by cumulative planimetry of their surface, the dissection and weighing method will therefore overestimate the actual IS, depending on the ratio of $x$ and d. The expected relation between ISDW and IS-CP would be: IS-DW $\approx$ $100 \bullet(1-2 x / d)+(2 x / d) \bullet$ IS-CP, with $2 x / d \rightarrow 0:$ IS-DW $\approx 100 \%$ (no tissue permeation) and with $2 x / d \rightarrow 1:$ IS-DW $\approx$ IS-CP (full tissue permeation) (Fig $5 B$ ). This emphasizes the importance of minimizing the slice thickness $d$ at a given and limited tissue permeation $x$ to ideally approach $\mathrm{d}=2 \mathrm{x}$.

Both of these effects, lesser discrimination due to limited precision during physical dissection as well as limited tissue permeation of TTC from the surface of the fresh heart slices, may therefore have affected the relation of IS-DW and IS-CP in our study to different degrees. While mere cross-contamination would have caused the regression line (continuous line in Fig 2) to cross the line of identity (broken line in Fig 2) at the $50 \%$ mark (Fig 5, panel $A$ ), merely limited permeation would have caused both lines to cross at the $100 \%$ mark (Fig 5, panel B). The fact that both lines intersect at $57.2 \%$, i.e. much closer to $50 \%$ than to $100 \%$, suggests that the deviation from the ideal in our study is due in large part to the limited precision of the manual dissection method and only partly due to the limited tissue permeation of TTC.

Additional regression analysis revealed that IS-CP, but not ISDW, correlated with return of mechanical function after I/R such as developed LVP, contractility and relaxation as well as metabolic function, i.e. myocardial oxygen consumption (Fig 4). Despite the lack of an independent method as a gold standard to compare the two TTC methods to in our study, this provides further evidence that the use of cumulative planimetry to measure IS in hearts of this size range may more appropriately reflect cardiac damage vs. protection than the manual dissection and weighing method can provide. Comparing function and tissue viability is naturally limited by the possibility of "stunning", i.e. the reversible reduction of cardiac function not accounted for by infarction. The results shown in Fig 4, however, render this possibility an unlikely factor in our study because the majority of all measurements do not show a combination of decreased function and low infarction. article is (C) Elsevier and permission has been granted for this version to appear in e-Publications@Marquette. Elsevier does not grant permission for this article to be further copied/distributed or hosted elsewhere without the express permission from Elsevier. 
Some limitations of our study have to be acknowledged. All hearts in this study were removed from the setup, cut into slices and then stained with TTC. Disadvantages of this order include a) limited tissue permeation of the TTC and $b$ ) the requirement of the fresh hearts to be cut immediately into slices of a minimum thickness in order to maintain their integrity during the cutting process. In fresh guinea pig hearts we found this thickness to be at least $3 \mathrm{~mm}$. The lack of adequate permeation of the dye using our current method implies that a more effective method to have deeper penetration of the dye would be to perfuse the TTC into the coronary circulation briefly, just before removing the heart. The heart will be frozen and then cut into thinner slices using an automated microtome. Furthermore, we only compared the two different methods of measuring and analyzing infarct size after TTC staining with each other. Though outside of the scope of our study, comparing them against a TTC-independent infarct size measurement may potentially lead to different results.

In conclusion, limited precision in the ability to manually dissect stained from unstained tissue and, to a lesser degree, limited tissue permeation when stained by diffusion in a TTC bath introduce biases in the assessment of myocardial IS when done by dissection and weighing and lead to an overestimation of infarct size. Therefore, cumulative planimetry appears to be superior and to more accurately reflect the degree of tissue damage.

\section{Acknowledgments}

The authors would like to thank James S. Heisner, B.S. (Research Technologist, Department of Anesthesiology, Medical College of Wisconsin, Milwaukee, Wisconsin, U.S.A.) for his valuable contributions to this study. Supported in part by grant No. $0735325 \mathrm{~N}$ from the American Heart Association (to Dr. Rhodes), and No. HL58691 (to Dr. Stowe) and No. HL 073246-01 (to Dr. Camara) from the National Institutes of Health (Bethesda, Maryland).

\section{Footnotes}

Conflict of Interest Statement: None of the authors has any potential conflicts of interests that may pertain to the present study. 
Publisher's Disclaimer: This is a PDF file of an unedited manuscript that has been accepted for publication. As a service to our customers we are providing this early version of the manuscript. The manuscript will undergo copyediting, typesetting, and review of the resulting proof before it is published in its final citable form. Please note that during the production process errors may be discovered which could affect the content, and all legal disclaimers that apply to the journal pertain.

\section{References}

1. Altman FP. Tetrazolium salts and formazans. Progress in Histochemistry and Cytochemistry. 1976;9:1-56.

2. Camara AK, Riess ML, Kevin LG, Novalija E, Stowe DF. Hypothermia Augments Reactive Oxygen Species Detected in the Guinea Pig Isolated Perfused Heart. American Journal of Physiology - Heart and Circulatory Physiology. 2004;286:H1289-H1299.

3. Chiari PC, Pagel PS, Tanaka K, et al. Intravenous emulsified halogenated anesthetics produce acute and delayed preconditioning against myocardial infarction in rabbits. Anesthesiology. 2004;101:11601166.

4. Fishbein MC, Meerbaum S, Rit J, et al. Early phase acute myocardial infarct size quantification: validation of the triphenyl tetrazolium chloride tissue enzyme staining technique. American Heart Journal. 1981;101:593-600.

5. Goldlust EJ, Paczynski RP, He YY, Hsu CY, Goldberg MP. Automated measurement of infarct size with scanned images of triphenyltetrazolium chloride-stained rat brains. Stroke. 1996;27:1657-1662.

6. Hausenloy DJ, Yellon DM. Preconditioning and postconditioning: united at reperfusion. Pharmacology \& Therapeutics. 2007;116:173-191.

7. Hindman BJ, Dexter F, Subieta A, Smith T, Cutkomp J. Brain injury after cerebral arterial air embolism in the rabbit as determined by triphenyltetrazolium staining. Anesthesiology. 1999;90:1462-1473.

8. Kersten JR, Schmeling TJ, Pagel PS, Gross GJ, Warltier DC. Isoflurane mimics ischemic preconditioning via activation of KATP channels: reduction of myocardial infarct size with an acute memory phase. Anesthesiology. 1997;87:361-370.

9. Khalil PN, Siebeck M, Huss R, et al. Histochemical assessment of early myocardial infarction using 2,3,5-triphenyltetrazolium chloride in blood-perfused porcine hearts. Journal of Pharmacological and Toxicological Methods. 2006;54:307-312. 
NOT THE PUBLISHED VERSION; this is the author's final, peer-reviewed manuscript. The published version may be accessed by following the link in the citation at the bottom of the page.

10. Murry $C E$, Jennings RB, Reimer KA. Preconditioning with ischemia: a delay of lethal cell injury in ischemic myocardium. Circulation. $1986 ; 74: 1124-1136$.

11. Riess ML, Camara AKS, Chen Q, et al. Altered NADH and improved function by anesthetic and ischemic preconditioning in guinea pig intact hearts. American Journal of Physiology - Heart and Circulatory Physiology. 2002;283:H53-60.

12. Riess ML, Camara AKS, Kevin LG, An J, Stowe DF. Reduced reactive $\mathrm{O}_{2}$ species formation and preserved mitochondrial $\mathrm{NADH}$ and $\left[\mathrm{Ca}^{2+}\right]$ levels during short-term $17^{\circ} \mathrm{C}$ ischemia in intact hearts. Cardiovascular Research. 2004;61:580-590.

13. Riess ML, Novalija E, Camara AK, et al. Preconditioning with sevoflurane reduces changes in nicotinamide adenine dinucleotide during ischemiareperfusion in isolated hearts: reversal by 5 -hydroxydecanoic acid. Anesthesiology. 2003;98:387-395.

14. Stowe DF, Camara AK, Heisner JS, Aldakkak M, Harder DR. Ten-hour preservation of guinea pig isolated hearts perfused at low flow with air-saturated Lifor solution at 26 \{degrees\}C: comparison to ViaSpan solution. American Journal of Physiology - Heart and Circulatory Physiology. 2007;293:H895-901. 